\title{
Carotenoids and antioxidant capacities from Canarium odontophyllum Miq. fruit.
}

\begin{abstract}
Carotenoids were isolated and identified from peel, pulp and seed fractions of Canarium odontophyllum Miq., and their antioxidant capacities were evaluated. all-trans- $\beta$-carotene was present in a large amount in peel $(69.5 \pm 1.0 \mathrm{mg} / \mathrm{kg})$, followed by pulp $(31.1 \pm 0.76$ $\mathrm{mg} / \mathrm{kg})$ and seed $(15.1 \pm 3.0 \mathrm{mg} / \mathrm{kg})$. Additionally, 15 -cis- $\beta$-carotene, 9 -cis- $\beta$-carotene and 13 -cis- $\beta$-carotenes were also major contributors to carotenoid contents in peel, pulp and seed fractions. Pulp exhibited excellent $\beta$-carotene bleaching activity, significantly higher than peel and seed; high 1,1-diphenyl-2-picrylhydrazyl (DPPH) radical-scavenging activity, whereas peel exhibited significantly higher scavenging activity of 2,2'-azino-bis(3ethylbenzthiazoline-6-sulphonic acid) (ABTS) radicals. All the extracts exhibited good inhibitory effect against hydrogen peroxide-induced haemoglobin oxidation, ranging from 45.3 to $59.7 \%$. This is the first report about carotenoids and antioxidant capacities from $\mathrm{C}$. odontophyllum fruit, and indicates that this fruit can be explored and promoted as a potential source of natural antioxidants.
\end{abstract}

Keyword: Antioxidant activity; Canarium odontophylluim; Carotenoids; Haemoglobin oxidation. 